\title{
Diagnostic accuracy of rapid urine dipstick test to predict urinary tract infection among pregnant women in Felege Hiwot Referral Hospital, Bahir Dar, North West Ethiopia
}

Tazebew Demilie', Getenet Beyene ${ }^{2,3}$, Selabat Melaku ${ }^{2,3}$ and Wondewosen Tsegaye ${ }^{1 *}$

\begin{abstract}
Background: Untreated bacteriuria during pregnancy has been shown to be associated with low birth-weight and premature delivery. Therefore, routine screening for bacteriuria is advocated. The decision about how to screen pregnant women for bacteriuria has always been a balance between the cost of screening versus the sensitivity and specificity. This study was designed to evaluate the diagnostic accuracy of the rapid dipstick test to predict urinary tract infection in pregnancy against the gold standard urine culture.

Method: A total of 367 mid stream urine samples were collected, inoculated on MacConkey, Manitol salt agar (MSA) and blood agar and incubated aerobically at $37^{\circ} \mathrm{C}$ for overnight. Specimens were classified as "positive" for urinary tract infection (UTI) if the growth of the pathogen(s) was at a count $\geq 10^{5}$ colony-forming units per milliliter (cfu/mL) of urine and classified as "negative" with growth of $<10^{5} \mathrm{cfu} / \mathrm{mL}$. Urine samples were tested for the presence of nitrite and leukocyte esterase using dipstick rapid test in accordance to the manufacturer's instructions.

Results: From the total study participants, 37 pregnant women were symptomatic and the remaining 330 pregnant women were asymptomatic. The sensitivity and specificity of dipstick tests of leukocyte esterase was $50 \%$ and $89.1 \%$ for pregnant women with asymptomatic UTI(ABU) and $71.4 \%$ and $86.7 \%$ for symptomatic UTI respectively and for nitrite $35.7 \%$ and $98.0 \%$ for ABU and $57.1 \%$ and $96.7 \%$ symptomatic UTI.

Conclusion: This study revealed that the use of dipstick leukocyte esterase and nitrite for screening UTI particularly asymptomatic bacteriuria was associated with many false positive and negative results when it was compared against the gold standard culture method. The low sensitivity and positive predictive value of urine dipstick test proved that culture should be used for the diagnosis of UTI.
\end{abstract}

Keywords: Dipstick test, Pregnant women, UTI, Asymptomatic bacteriuria

\section{Background}

Urinary tract infection (UTI) is an infection caused by the presence and growth of microorganisms anywhere in the urinary tract. It is usually happen due to bacteria from the digestive tract ascending the opening of the urethra and begin to multiply to cause infection [1,2]. Pregnant women are at increased risk for UTIs, beginning in week

\footnotetext{
* Correspondence: wondewosentsg@gmail.com

'Department of Microbiology, Immunology and Parasitology, College of Medicine and Health Sciences, Bahir Dar University, P.O.Box 79, Bahir Dar, Ethiopia

Full list of author information is available at the end of the article
}

6 and peaking during weeks 22 to 24, approximately 90 percent of pregnant women develop urethral dilatation, which will remain until delivery. Increased bladder volume and decreased bladder tone, along with decreased urethral tone, contribute to increased urinary stasis and ureterovesical reflux [3].

UTI can be either symptomatic or asymptomatic, and patients with significant bacteriuria who have symptoms referable to the urinary tract are said to have symptomatic bacteriuria. Asymptomatic bacteriuria (ABU) is a condition characterized by bacteriuria without classical symptoms attributable to the urinary tract [4]. The relatively 
high prevalence of UTI particularly asymptomatic bacteriuria during pregnancy, which will have significant consequences can be avoided with treatment if they are screened early for bacteriuria. The decision about how to screen asymptomatic women for bacteriuria has always been a balance between the cost of screening versus the sensitivity and specificity [5]. In addition, the correctness of the laboratory test in determining the individual status is crucial not to give false positive or negative results that may lead to unnecessary prescription of drug or inappropriate management.

Even though there are various methods for screening, urine culture still remains the gold standard for diagnosis of UTI and has been advocated as a screening procedure for urinary tract infection in pregnancy. It is however, expensive, and takes 24-48 h to obtain result and requires skilled personnel. Unfortunately, appropriate investigations necessary for the diagnosis of urinary tract infection are not available in many health facilities [6].

Dipstick test is one of the qualitative diagnostic method used to detect UTI and have the advantage of being easy to perform, interpret, can be carried out in primary care giving facilities and result can be obtained immediately [7]. Though evaluation of dipstick test has been conducted in different countries $(6,7)$, it has never been tested in the northern part of Ethiopia around Bahir Dar and information generated from this study will help clinician and laboratory technologist for the decision to determine to use culture and dipstick test in accordance their relevance. Thus, the objective of this study was to evaluate the diagnostic accuracy of the rapid dipstick test to predict urinary tract infection in pregnancy in comparison with the gold standard of urine culture.

\section{Methods}

A hospital based prospective cross sectional study was conducted at Felege Hiwot Referral Hospital (FHRH) from October 2010 to January 2011 to evaluate the diagnostic accuracy of the rapid urine dipstick test to predict urinary tract infection in pregnancy with age group ranged from 17 to 40 years with gestation period of 16 to 38 weeks coming for prenatal follow up.

A pre-designed and structured questionnaire was used for the collection of data on socio-demographic characteristics. The presences of symptoms of UTI were checked by Gynaecologists and senior nurses.

A total of 367 pregnant women (37 with complaints and 330 without complaints of UTI) were participated in the study and pregnant women who have taken antibiotics within seven days at the time of recruitment and who were not willing to participate were excluded from this study.

Symptomatic UTI refers to patients whose urine is yielding positive culture $\left(\geq 10^{5} \mathrm{cfu} / \mathrm{ml}\right)$ and who have symptoms referable to the urinary tract infection, where as asymptomatic bacteriuria (ABU) refers to pregnant women whose two consecutive urine samples showed positive cultures $\left(\geq 10^{5} \mathrm{cfu} / \mathrm{ml}\right)$ of the same uropathogen and without showing sign and symptoms of UIT [8].

\section{Specimen collection and processing}

To reduce the risk of contamination, pregnant women were informed to clean their hands with water and their genital area with swab soaked in normal saline before collection of the clean catch mid stream urine samples.The samples were collected using sterile, wide mouthed glass bottles with screw cap tops and were processed in the laboratory within 2 hours of collection and specimens that were not processed within 2 hours were kept refrigerated at $4^{\circ} \mathrm{C}$ until it was processed.

\section{Dipstick test}

From all participants urine samples were tested for the presence of nitrite and leukocyte esterase using dipstick rapid test. The Ames Multiple Reagent Strip (Bayer Diagnostics Australia) was used for all specimens in accordance with the manufacturer's instructions. A positive dipstick was defined as the presence of nitrites or a reaction of greater than or equal to a trace of leukocytes. A negative dipstick was defined as the absence of any reaction for leukocytes and nitrites.

Urine dipstick bottles were stored at room temperature and expire dates also checked before use. Urine dipstick test results were interpreted according to the manufacturer's instruction.

\section{Quantitative urine culture}

Mixed freshly collected clean catch mid stream urine specimens were taken using a sterile calibrated wire loop (one that hold $1 / 500 \mathrm{ml}$ or $0.002 \mathrm{ml}$ ) and were inoculated on a plate MacConkey (Oxoid, Ltd, England), Manitol salt agar (MSA) (Oxoid, Ltd, England) and blood agar (Oxoid, Ltd, England) and were incubated aerobically at $37^{\circ} \mathrm{C}$ for overnight. Specimens were classified as "positive" for UTI if the yielded of growth of a pathogen(s) was at a count $\geq$ $10^{5}$ colony-forming units per milliliter $(\mathrm{cfu} / \mathrm{mL})$ of urine. Specimens were classified as "negative" if culture showed growth of $<10^{5} \mathrm{cfu} / \mathrm{mL}$ and considered as contaminated, when mixed bacterial growth was observed $[2,8]$.

Standard reference strains, Staphylococcus aureus (ATCC25923), Escherichia coli (ATCC25922 and P. aeruginosa (ATCC 27853) were used to assure testing of quality of culture media.

\section{Data entry and analysis}

All data were entered into the computer and analyzed using the EPI Info Software. The degree of correlation between the components of the dipstick singly and in 
various combinations was compared against urine culture result. The comparative diagnostic value of the various components of the dipstick test and culture were evaluated in terms of sensitivity, specificity, positive predictive value (PPV) and negative predictive value (NPV) against urine culture (gold standard).

\section{Ethical consideration}

The study was conducted after getting a full approval from Jimma University post graduate and research coordinating office, Amhara national regional state health bureau and FHRH. In addition written informed consent for the study was obtained from the study participants and confidentiality of results was kept. All dipstick and culture results were sent to the responsible physicians as soon as possible so that the pregnant women were directly benefited from the study.

\section{Results}

A total of 367 pregnant women with and without symptoms of urinary tract infection (UTI) were investigated during the study period. The minimum and the maximum age of the pregnant women were 17 and 39 years respectively with mean age was 25.8 . The predominated age group was $20-24$ years (43.9\%), the majority group of study participants are at their second (39.1\%) and third $(47.0 \%)$ stage of pregnancy with no significance variation with the rate of bacteriuria . Educational status of participants varied from illiterate to postgraduate studies, $66(18 \%)$ of the respondents were unable to read and write while $25.1 \%$ of the participants had higher education (Table 1).

Out of the total 367 study participants, 330 pregnant women were without sign and symptoms for UTI and were screened for asymptomatic bacteriuria (ABU). The dipstick rapid test result showed that 55 (15\%) of the pregnant women were positive for leukocyte esterase and 21 (5.7\%) pregnant women were positive for nitrite (Table 2).

From the 35 bacterial isolates ( 28 from $\mathrm{ABU}$ ), 23 were Gram negative while 12 were Gram positive bacteria. Among the identified bacteria E.coli was the most common bacteria accounting for 16 (45.7\%) isolates followed by Coagulase negative Staphylococcus (CNS) and S.aureus with isolation rate of $17.1 \%$ and $8.6 \%$ respectively, and only 2 (5.7\%) Proteus mirabilis were indentified (data not shown).

Taking culture result as the gold standard, the sensitivity and specificity was $50 \%$ and $89.1 \%$, and $35.7 \%$ and $98.0 \%$ for leukocyte esterase and nitrite respectively for asymptomatic UTI (Table 2).

When we consider both leukocyte esterase and nitrite dipstick test result together taking account positive results in either of the test, the sensitivity of dipstick was increased to $71.4 \%$ than using either of the tests alone.
Table 1 Socio-demographic characteristics of study participants in Felege Hiwot Referral Hospital (FHRH), Bahir Dar, October 2010-January 2011

\begin{tabular}{|c|c|c|}
\hline Variables & Number & Percent \\
\hline \multicolumn{3}{|l|}{ Age } \\
\hline $15-24$ & 200 & 54.5 \\
\hline $25-34$ & 159 & 43.3 \\
\hline $35-44$ & 8 & 2.2 \\
\hline \multicolumn{3}{|l|}{ Gestation stage } \\
\hline First trimester & 49 & 13.4 \\
\hline Second trimester & 141 & 38.4 \\
\hline Third trimester & 177 & 48.2 \\
\hline \multicolumn{3}{|l|}{ Marital status } \\
\hline Married & 334 & 99.2 \\
\hline Others & 3 & 0.8 \\
\hline \multicolumn{3}{|l|}{ Occupation } \\
\hline Employed & 110 & $3 c$ \\
\hline Unemployed & 257 & 7 \\
\hline \multicolumn{3}{|l|}{ Residence } \\
\hline Urban & 354 & 96.5 \\
\hline Rural & 13 & 3.5 \\
\hline \multicolumn{3}{|l|}{ Religion } \\
\hline Orthodox & 321 & 87.5 \\
\hline Muslim & 33 & 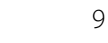 \\
\hline Protestant & 13 & 3.5 \\
\hline \multicolumn{3}{|l|}{ Ethnicity } \\
\hline Amhara & 357 & 97.3 \\
\hline Oromo & 5 & 1.2 \\
\hline Tigray & 3 & 0.8 \\
\hline Other & 2 & 0.5 \\
\hline \multicolumn{3}{|l|}{ Education } \\
\hline Illiterate & 66 & 18 \\
\hline Read and write & 12 & 3.3 \\
\hline Elementary & 67 & 18.3 \\
\hline High school & 130 & 35.4 \\
\hline Higher education & 92 & 25 \\
\hline \multicolumn{3}{|l|}{ Income/month } \\
\hline$<500$ & 53 & 14.4 \\
\hline $501-1000$ & 96 & 27.5 \\
\hline $1001-1500$ & 63 & 17. \\
\hline $1501-2000$ & 55 & 15.0 \\
\hline$>2000$ & 100 & 28. \\
\hline Total & 367 & \\
\hline
\end{tabular}

Where as if we take only positive cases for both tests the sensitivity were decreased to $22.9 \%$ than either of the tests alone. 
Table 2 Dipstick test result for leukocyte esterase and nitrite in relation with culture result of ABU, symptomatic UTI and overall UTI in FHRH, Bahirdar, October 2010-January 2011

\begin{tabular}{|c|c|c|c|c|c|c|c|c|c|}
\hline \multirow[t]{2}{*}{ Type of UTI } & \multirow[t]{2}{*}{ Dipstick tests } & & \multicolumn{3}{|c|}{ Culture } & \multirow[t]{2}{*}{ Sensitivity } & \multirow[t]{2}{*}{ Specificity } & \multirow[t]{2}{*}{ PPV } & \multirow[t]{2}{*}{ NPV } \\
\hline & & & Positive $\mathrm{N}^{\circ}(\%)$ & Negative $\mathbf{N}^{\circ}(\%)$ & Total $\mathrm{N}^{\circ}(\%)$ & & & & \\
\hline \multirow[t]{6}{*}{$A B U$} & LE & Positive & 14(29.9) & $33(70.2)$ & $47(14.2)$ & $50.0 \%$ & $89.1 \%$ & $29.8 \%$ & $95.1 \%$ \\
\hline & & Negative & $14(5)$ & 269(95) & 283(85.8) & & & & \\
\hline & & Total & $28(8.5)$ & $302(91.5)$ & $330(100)$ & & & & \\
\hline & Nitrites & Positive & $10(62.5)$ & $6(37.5)$ & $16(4.9)$ & & & & \\
\hline & & Negative & $18(5.7)$ & 296(94.3) & $314(95.1)$ & $35.7 \%$ & $98 \%$ & $62.5 \%$ & $94.3 \%$ \\
\hline & & Total & $28(8.5)$ & $302(91.5)$ & $330(100)$ & & & & \\
\hline \multirow[t]{6}{*}{ Symptomatic UTI } & LE & Positive & $5(62.5)$ & $3(37.5)$ & $8(21.6)$ & & & & \\
\hline & & Negative & $2(6.9)$ & 27(93.1) & $29(78.4)$ & $71.4 \%$ & $90.0 \%$ & $62.5 \%$ & $93.1 \%$ \\
\hline & & Total & $7(18.9)$ & $30(81.1)$ & $37(100)$ & & & & \\
\hline & Nitrites & Positive & $4(80)$ & $1(20)$ & $5(13.5)$ & & & & \\
\hline & & Negative & $3(9.4)$ & 29(90.6) & $32(86.5)$ & $57.1 \%$ & $96.7 \%$ & $80.0 \%$ & $90.6 \%$ \\
\hline & & Total & $7(18.9)$ & $30(81.1)$ & $37(100)$ & & & & \\
\hline \multirow[t]{12}{*}{ Overall UTI } & LE & Positive & $18(32.7)$ & $37(67.3)$ & $55(15.0)$ & & & & \\
\hline & & Negative & $17(5.5)$ & 295(94.5) & $312(85)$ & $51.4 \%$ & $88.9 \%$ & $32.7 \%$ & $94.5 \%$ \\
\hline & & Total & $35(9.5)$ & $332(90.5)$ & $367(100)$ & & & & \\
\hline & Nitrites & Positive & 15(71.4) & $6(28.6)$ & $21(5.7)$ & & & & \\
\hline & & Negative & $20(5.8)$ & $326(94.2)$ & $346(94.3)$ & $42.9 \%$ & $98.2 \%$ & $71.4 \%$ & $94.2 \%$ \\
\hline & & Total & $35(9.5)$ & $332(90.5)$ & $367(100)$ & & & & \\
\hline & LE or Nitrites & Positive & $25(71.4)$ & 10(26.6) & $35(9.5)$ & & & & \\
\hline & & Negative & 10(3.0) & $322(97)$ & $332(90.5)$ & $71.4 \%$ & $97.0 \%$ & $71.4 \%$ & $97.0 \%$ \\
\hline & & Total & $35(9.5)$ & $332(90.5)$ & $367(100)$ & & & & \\
\hline & LE and nitrites & Positive & $8(72.7)$ & $3(27.3)$ & $11(3.0)$ & & & & \\
\hline & & Negative & $27(7.6)$ & $329(92.4)$ & $356(97)$ & $22.9 \%$ & $99.1 \%$ & $72.7 \%$ & $92.4 \%$ \\
\hline & & Total & $35(9.5)$ & $332(90.5)$ & $367(100)$ & & & & \\
\hline
\end{tabular}

key: ABU: Asymptomatic Bacteruria, LE: Leukocyte Esterase, NPV: Negative predictive value, PPV: Positive predictive value, UTI: Urinary tract infection.

\section{Discussion and conclusions}

Routine screening pregnant women for bacteriuria advocated since untreated bacteriuria during pregnancy has been shown to be associated with low birth-weight and premature delivery [9].

In our study the sensitivity and specificity of urine dipstick (nitrate and leukocyte esterase in combination) was $22.9 \%$ and $99.1 \%$ respectively which is comparable with the study done by Masinde et al. [10] in Tanzania, who reported the sensitivity and specificity of the dipstick test result $38.9 \%$ and $86.7 \%$ respectively. In the current study the sensitivity of leukocyte esterase and nitrite was $51.4 \%$ and $42.9 \%$, while its specificity was $88.9 \%$ and $98 \%$ which has similar pattern with the study reported by Kacmaz et al. [6] where the sensitivity of urine leukocyte esterase and nitrite was found to be $70 \%$ and $60 \%$, and specificity was $92.5 \%$ and $99.2 \%$ respectively.

In our study the sensitivity of leucocytes esterase for detection $\mathrm{ABU}$ was $50 \%$ where as the sensitivity for nitrate was $35.7 \%$ which is comparable with a study on diagnostic accuracy of dipstick for the diagnosis of $\mathrm{ABU}$ in Nigeria by Eigbefoh et al. [11] who reported that the sensitivity of leucocytes esterase for detecting ABU was $56.8 \%$ and the sensitivity of nitrite was $21.6 \%$.

This study witnessed that sensitivity of urine dipstick for nitrite and leukocyte esterase slightly increases in symptomatic UTI than ABU. A study by Assefa et al. [12] also proved that percentage of significant pyuria slightly increase in symptomatic UTI than ABU. The sensitivity of leukocyte esterase and nitrite increase when taking positive result when one of the test was positive, while the sensitivity decrease when considering positive result when in both tests were positive. A study by Eigbefoh et al. [11] in Nigeria also reported decrease in the sensitivity of urine dipstick when both leukocyte esterase and nitrite test result were used together.

In Ethiopia the majority of health care facilities use dipstick analysis for nitrites and leukocyte esterase determination and microscopic examination for pyuria and bacteriuria for screening UTI (both ABU and symptomatic 
UTI) but according to different studies these dipstick tests have poor sensitivity and positive predictive values to detect bacteriuria especially in asymptomatic pregnant women $[5,13,14]$. In this study, it is also shown that urine dipstick testing for nitrites and leukocyte esterase can poorly detect all the culture positive bacteriuria cases in pregnant women. The low sensitivity of leukocyte esterase may be attributed to that the presence of leukocyturia does not always correlate with bacteriuria. Pyuria is not specific for UTI and may occur with other inflammatory disorders of the genitourinary tract (e.g., vaginitis) and infections with other uropathogens such as Chlamydia and N.gonorrhoeae. Moreover, the leukocyturia may continue even if the bacteriuria has cleared spontaneously or after treatment $[6,15,16]$. In contrast the low sensitivity of nitrite may be due to that nitrite test depends on the detection of nitrite in the urine which is formed from nitrate by many uropathogens. Positive nitrite test indicates that nitrite has been produced from the reduction of nitrate by enteric bacteria, most commonly by the genera of the $E n$ terobacteriaceae family. The presence of nitrite is highly specific for bacteriuria, but several uropathogens other than Enterobacteriaceae do not reduce nitrate to nitrite $[6,17]$. False negative results may occur when a UTI is caused by organism that do not contain nitrite reductase, when the urine has been in the bladder for insufficiently long periods for the reduction of nitrite to occur or when dietary nitrite is absent. A negative test may also signify absence of nitrites in the urine due to a reduction of nitrates beyond the nitrite stage $[6,14]$.

The use of dipstick leukocyte esterase and nitrite for screening UTI particularly with asymptomatic bacteriuria was associated with many false positive and negative results as compared to the gold standard culture method. Despite these limitations, a positive test suggestive of UTI for further evaluation of clinical manifestations for empirical treatment, while a negative test is an indication for urine culture. The urine dipstick test, if positive, will also be useful in follow-up of patient after treatment of urinary tract infection. This is useful in poor resource setting especially in the third world where there is a dearth of trained personnel and equipment for urine culture. Though this study proved the low sensitivity and specificity of the dipstick tests, the tests can be used to manage at least positive cases empirically.

\section{Competing interests}

The authors declare that they have no competing interests.

\section{Authors' contributions}

DT was responsible for inception of the idea and write up of the proposal and conduct the laboratory work. BG was responsible in designing the study and drafting the manuscript, whereas TW was involved in the design of study the analysis and interpretation of the data, and correction of the final draft of the manuscript.MS was responsible for collection of samples and data. All authors read and approved the final manuscript.

\section{Acknowledgements}

We would like to extend our thanks to Jimma University for financially supporting the research and study participants for their participation, and Bahir Dar Regional Laboratory for providing us facilities of laboratory to conduct the tests.

\section{Author details}

'Department of Microbiology, Immunology and Parasitology, College of Medicine and Health Sciences, Bahir Dar University, P.O.Box 79, Bahir Dar, Ethiopia. ${ }^{2}$ Department of Laboratory Sciences and Pathology, College of Public Health and Medical Sciences, Jimma University, P.O Box 378, Jimma, Ethiopia. ${ }^{3}$ Microbiologist in Bahir Dar regional laboratory, Regional Health Research Laboratory, Microbiologist in Bahir Dar University, Bahir Dar, Northwest Ethiopia, Ethiopia.

Received: 14 September 2013 Accepted: 16 July 2014

Published: 29 July 2014

\section{References}

1. Rahimkhani M, Khavari-Daneshvar H, Sharifian R: Asymptomatic bacteriuria and Pyuria in pregnancy. Acta Medica Iran 2008, 46:409-412.

2. Okonko IO, ljandipe LA, llusanya OA, Donbraye-Emmanuel OB, Ejembi J, Udeze O, Egun OC, Fowotade A, Nkang AO: Incidence of urinary tract infection (UTI) among Pregnant women in Ibadan, South-Western Nigeria. Afr J Biotechnol 2009, 8:6649-6657.

3. Moghadas AJ, Irajian G: Asymptomatic urinary tract infection in pregnant women. Iranian J Pathol 2009, 4:105-108.

4. Ethel S, Bhat GK, Hegde BM: Bacterial adherence and humoral immune response in women with symptomatic and asymptomatic urinary tract infection. Indian J Med Microbio 2006, 24:30-33.

5. Loh KY, Sivalingam N: Urinary tract infections in pregnancy. Malaysian Fam Physician 2007, 2:54-57.

6. Kacmaz B, Cakir O, Biri A: Evaluation of rapid urine screening tests to detect asymptomatic bacteriuria in pregnancy. Jpn J Dis 2006, 59:261-263.

7. Whiting P, Westwood M, Watt I, Cooper J, Kleijnen J: Rapid tests and urine sampling techniques for the diagnosis of urinary tract infection (UTI) in children under five years: a systematic review. BMC Pediatric 2005, 5:1-13.

8. Khatun S, Nessa A, Mahmood A: Urinary tract infections in pregnancy. ORION 1999, 4:15-20.

9. Romero R, Oyarzun E, Mazor M, Sirtori M, Hobbins JC, Bracken M: Meta-analysis of the relationship between asymptomatic bacteriuria and preterm delivery/low birth weight. Obstet Gynecol 1989, 73:576-582.

10. Masinde A, Gumodoha B, Kilonzo A, Mshana SE: Prevalence of urinary tract infection among women at Bugando Medical Center, Mwanza, Tanzania. Tanzania J Health Res 2009, 11:154-159.

11. Eigbefoh JO, Isabu P, Okpere E, Abebe J: The diagnostic accuracy of the rapid dipstick test to predict asymptomatic urinary tract infection of pregnancy. J Obstet Gynaecol 2008, 28:490-495.

12. Assefa A, Asrat D, Woldeamanuel $Y, G /$ Hiwot $Y$, Abdella A, Melesse T: Bacterial profile and drug susceptibility pattern of urinary tract infection in pregnant women at Tikur Anbessa Specialized Hospital Addis Ababa, Ethiopia. Ethiop Med J 2008, 46:227-235.

13. Hazhir S: Bacteriuria in pregnant women. Urol J (Tehran) 2007, 4:24-27.

14. Garingalo-Molina FD: Asymptomatic bacteriuria among pregnant women: overview of diagnostic approaches. Phil J Microbiol Infect Dis 2000, 29:177-186

15. Colgan R, Nicolle LE, Mcglone A, Hooton TM: Asymptomatic bacteriuria in adults. Am Fam Physician 2006, 74:985-990.

16. Franz $\mathrm{M}$, Horl WH: Common errors in diagnosis and management of urinary tract infection, Pathophysiologic and pathologic techniques. Nephrol Dial Transplant 1999, 14:2746-2753.

17. Patel HD, Livsey SA, Swann RA, Bukhari SS: Can urine dipstick testing for urinary tract infection at point of care reduce laboratory workload? J Clin Pathol 2005, 58:951-954.

doi:10.1186/1756-0500-7-481

Cite this article as: Demilie et al: Diagnostic accuracy of rapid urine dipstick test to predict urinary tract infection among pregnant women in Felege Hiwot Referral Hospital, Bahir Dar, North West Ethiopia. BMC Research Notes 2014 7:481. 